\title{
Vitamin D status in chronic obstructive pulmonary disease
}

\author{
F. R. Baldrick ${ }^{1}$, J. S. Elborn ${ }^{2,3}$, J. V. Woodside ${ }^{1}$, K. Treacy ${ }^{2}$, J. Bradley ${ }^{2}$, B. Schock ${ }^{3}$, M. Ennis ${ }^{3}$, \\ K. Dollin ${ }^{1}$, A. McGinty ${ }^{1}$, I. S. Young ${ }^{1}$ and M. C. McKinley ${ }^{1}$ \\ ${ }^{1}$ Centre for Public Health, Queen's University Belfast, BT12 6BJ, ${ }^{2}$ Respiratory Medicine Group, Belfast City Hospital, \\ $B T 97 A B, U K$ and ${ }^{3}$ Centre for Infection and Immunity, Queen's University Belfast, BT9 7BL, UK
}

Vitamin D is well known for its role in bone health and calcium homeostasis. However, emerging evidence suggests that vitamin D may also have an influence on other health issues such as type 2 diabetes, cardiovascular disease, cancer, autoimmune diseases and respiratory function $^{(1,2)}$. Chronic obstructive pulmonary disease (COPD) is characterised by airflow limitation and is associated with an abnormal inflammatory response ${ }^{(3)}$. It has been hypothesised that vitamin D deficiency may enhance chronic airway and systemic inflammation and increase the risk of infectious exacerbations ${ }^{(1)}$ in COPD. Vitamin D status in this population is, therefore, of interest. The aim of the present study was to examine the prevalence of sub-optimal vitamin D status in a sample of patients with moderate to severe COPD and to compare the vitamin D status of a sub-sample of this patient group with healthy age-, sex-, and season-matched controls.

The serum 25-hydroxyvitamin D (25-OHD) levels of 81 Northern Irish patients with moderate to severe COPD (classified according to Global Initiative for Chronic Obstructive Lung Disease (GOLD) guidelines ${ }^{(3)}$ ) were measured using Ultra Performance Liquid Chromatography followed by tandem mass spectrometry (UPLC/MS/MS). Twenty-two COPD patients were matched for age-, sex-, and season of blood sample collection with healthy participants recruited from Northern Ireland at the same time as the COPD cohort.

The mean vitamin D status for male and female COPD patients was 35.3 (SD 23.3) nmol/l and 38.5 (SD 49.6) nmol/1, respectively $(P=0.701$; Independent samples t-test). There was no significant difference in mean vitamin $\mathrm{D}$ status between those with moderate (32.3 (sD 20.2) nmol/l) compared to severe COPD (39.8 (sD 44.8) nmol/l) $(P=0.372$; Independent samples t-test). Season of blood sample collection had no significant effect on vitamin D status in this group of COPD patients $(P=0.116$; analysis of variance). Overall, almost half of the COPD patients $(45.7 \%)$ were vitamin D deficient $(<25 \mathrm{nmol} / \mathrm{l})$, with $79 \%$ showing biochemical evidence of sub-optimal vitamin D status $(<50 \mathrm{nmol} / \mathrm{l})$. Only $10 \%$ of this sample had adequate vitamin D concentrations $(>75 \mathrm{nmol} / \mathrm{l})$. The vitamin $\mathrm{D}$ status of patients with moderate to severe $\operatorname{COPD}(n=22)$ was significantly lower compared with healthy age-, sex-, and season-matched controls $(n=22)(36.7$ (sD 36.8) nmol/l versus 52.5 (SD 23.2) nmol/l, respectively; $P=0.003$; Independent samples t-test).

Suboptimal vitamin D status was prevalent in this sample of patients with moderate to severe COPD. Furthermore, patients with moderate to severe COPD had significantly lower vitamin D concentrations compared to healthy age-, sex-, and season-matched controls. The potential role of vitamin D supplementation in the prevention or management of COPD is worthy of further investigation.

1. Janssens W, Lehouck A, Carremans C et al. (2009) Am J Resp Crit Care Med 179, 630-636.

2. Gilbert CR, Arum SM \& Smith CM (2009) J Can Thorac Soc 16, 75-80.

3. Global Initiative for Chronic Obstructive Lung Disease (GOLD) guidelines. (2001) NHLBI/WHO Workshop Report. 